\title{
a.m. \\ Proposed mechanism in cancer cure claim. The lipid connection
}

\section{Letter to Editor}

The purpose on my letter is to add a brief history behind a "cure for cancer" claim theory as proposed by Professor Hague in his recently published opinion in your journal. ${ }^{1}$ I should add that I concur that it could work in solid tumors. There are several reasons for the information expressed in this letter, which I would like to share with Dr Hague and your readers as follows:

The concept of an electrical mechanism towards cancer origin was first published by Dr. Denham Hartman in 1956 by, introducing a theory stating that "there are good reasons for assuming that endogenous irradiation in the living cells could lead to cancer via an obscure mechanism." "In an attempt to shed some light on that mechanism, in 2016 a Biophysical Hypothesis attributing cancer origin to a biophysically induced phenomenon occurring during cell respiration was published; ${ }^{3}$ subsequently the secondary role of UV light in swimmers melanoma genesis was proposed; again a biophysical mechanism arising from the breakdown of reactive oxygen species, such as $\mathrm{H}_{2} \mathrm{O}_{2}$ was rationalized as an additional factor in this type of Cancer Genesis.

In his opinion, Dr. Hague states, "We know have a good working theory to explain how Cell Sonics VIPP (read very intense pressure pulses) works in cancer". As an addition to his working theory, I would like to add a recently published biophysical mechanism describing the role of shockwaves in altering lipid droplets and the affected droplets in cancer etiology $y^{5}$. VIPP could possibly work in interfering with
Volume 10 Issue $3-2019$

\author{
Abraham A Embi \\ Citizen Scientist, USA
}

Correspondence: Abraham A Embi, Formerly Affiliated with VA Hospital, University of Miami, I 3442 SW 102 Lane, Miami, Florida, USA, 33 I86, Tel +305 505 4979, Email embi2 I@att.net

Received: May 02, 2019 | Published: May 09, 2019

cancer cells genesis, growth and proliferation as follows:

"It is known that shockwaves arising from ROS breakdown create oxygen bubbles, that these bubbles rupture and emit energy in the form of shockwaves. That when a surge of electrons reach a lipid droplet energy transfer ensues. Presented herein is the luminescence phenomenon (read static electricity) seen in lipid droplets once an energy saturation point is achieved....".

In support of the above statement, there is also published evidence where in plants, wounding triggered lipid droplets luminescence. ${ }^{6}$ In other words, in eukaryotes (read plants and animals) intracellular lipid organelles are damaged by energy from shockwaves; therefore it makes sense that by blocking this energy VIPP could possibly arrest cancer genesis and/or proliferation in its track (See Supplementary Attachment).

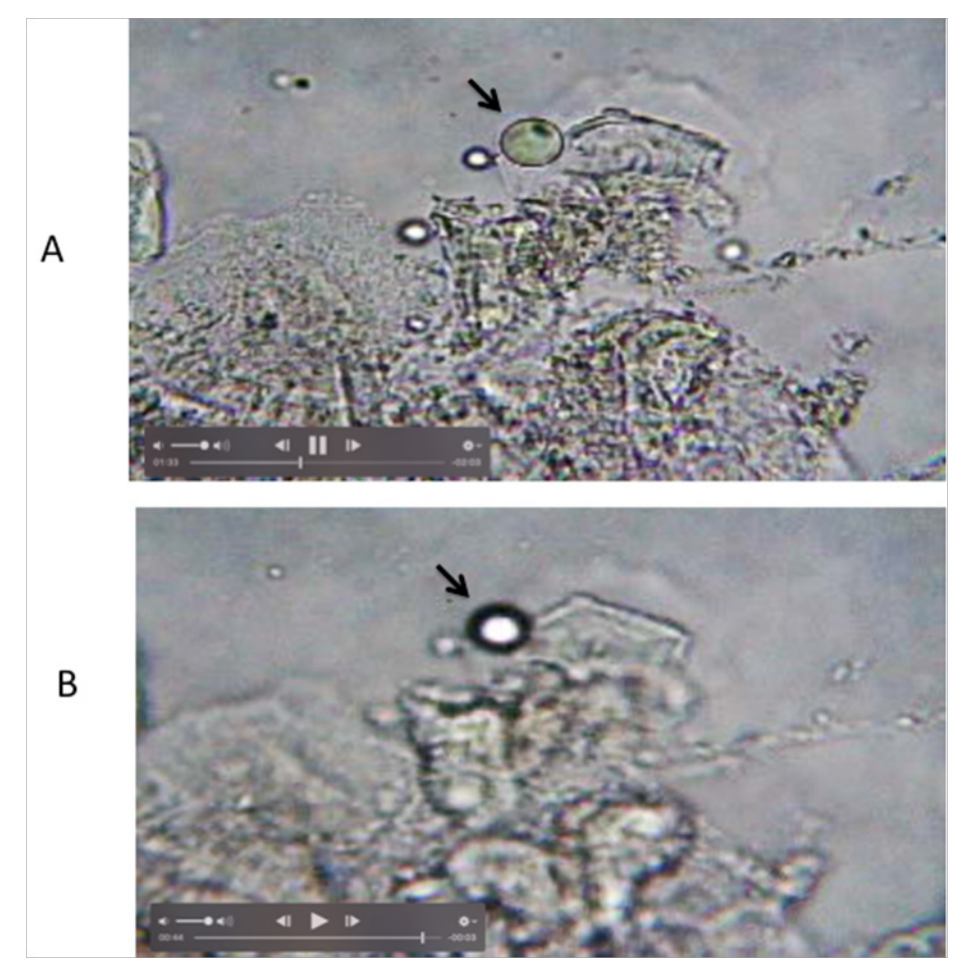

Figure I Additional demonstration of lipid droplet luminescence.

Panel $A=$ Black arrow pointing at lipid droplet control.

Panel $B=$ Showing lipid droplet luminescence. 
Notice the sudden burst of light causing the image to be out of focus. Notice in panel A smaller lipid droplets that were already in a luminescent phase.

For additional details, please link to: https://youtu.be/ pVYQD64PB-o

Reproduced from: Embi AA (2019) Introducing In Vitro Experiments of Oxygen Bubbles Shockwaves Triggering Intracellular Lipids Luminescence: Implications in Cancer Etiology. IJGR March 2019 (In Print)

For video recording please scan with phone QR Code below:

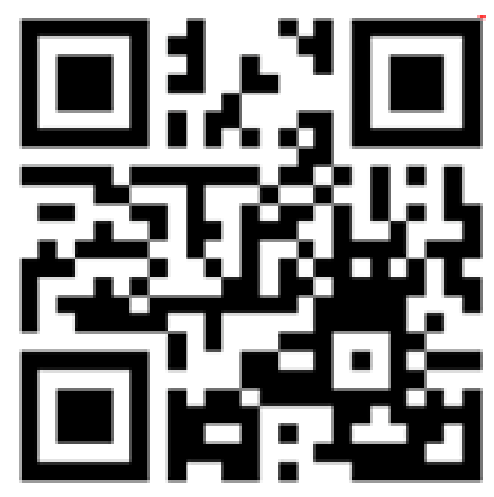

\section{Acknowledgments}

None.

\section{Conflict of interest}

The authors declare no conflict of interest.

\section{References}

1. Hague A. How the cure for cancer was discovered. J Cancer Prev Curr Res. 2019;10(1):13.

2. Harman D. Aging: a theory based on free radical and radiation chemistry. $J$ Gerontol. 1956;11(3):298-300.

3. Embi AA. Endogenous electromagnetic forces emissions during cell respiration as additional factor in cancer origin. Cancer Cell Int. 2016;28(16):60.

4. Embi AA. The secondary role of UV light in swimmers melanoma genesis. Int J Mol Biol Open Access. 2018;3(3):119-121.

5. Embi AA. Introducing In Vitro Experiments of Oxygen Bubbles Shockwaves Triggering Intracellular Lipids Luminescence: Implications n Cancer Etiology. International Journal of Research Granthaalayah. 2019;7(4):355-364.

6. Birtić S, Ksas B, Genty B, et al. Using spontaneous photon emission to image lipid oxidation patterns in plant tissues. Plant J. 2011;67(6):11031115 . 